\title{
Modified Gravity (MOG) fits to observed radial acceleration of SPARC galaxies
}

\author{
M. A. Green ${ }^{\mathrm{a}}$, J. W. Moffat ${ }^{\mathrm{a}, \mathrm{b}}$ \\ ${ }^{a}$ Perimeter Institute for Theoretical Physics, Waterloo ON N2L 2Y5, Canada \\ ${ }^{b}$ Department of Physics and Astronomy, University of Waterloo, Waterloo ON N2L 3G1, Canada
}

\begin{abstract}
The equation of motion in the generally covariant modified gravity (MOG) theory leads, for weak gravitational fields and nonrelativistic motion, to a modification of Newton's gravitational acceleration law. In addition to the metric $g_{\mu \nu}$, MOG has a vector field $\phi_{\mu}$ that couples with gravitational strength to all baryonic matter. The gravitational coupling strength is determined by the MOG parameter $\alpha$, while parameter $\mu$ is the small effective mass of $\phi_{\mu}$. The MOG acceleration law has been demonstrated to fit a wide range of galaxies, galaxy clusters and the Bullet Cluster and Train Wreck Cluster mergers. For the SPARC sample of rotationally supported spiral and irregular galaxies, McGaugh et al. [24] (MLS) have found a radial acceleration relation (RAR) that relates accelerations derived from galaxy rotation curves to Newtonian accelerations derived from galaxy mass models. Using the same SPARC galaxy data, mass models independently derived from that data, and MOG parameters $\alpha$ and $\mu$ that run with galaxy mass, we demonstrate that adjusting galaxy parameters within \pm 1 -sigma bounds can yield MOG predictions consistent with the given rotational velocity data. Moreover, the same adjusted parameters yield a good fit to the RAR of MLS, with the RAR parameter $a_{0}=(5.4 \pm .3) \times 10^{-11} \mathrm{~m} / \mathrm{s}^{2}$
\end{abstract}

\section{Introduction}

[The pioneering research by Zwicky [1] and Vera Rubin and collaborators [2, 3] showed an apparent discrepancy between the dynamics of galaxies and galaxy clusters and the predictions of Newtonian and Einstein gravity. The dynamical masses inferred from observations of galaxies and clusters were found to exceed the baryon mass in these systems. The existence of mysterious dark matter, forming halos around galaxies, was proposed to resolve the discrepancy. To date, there is no convincing evidence in deep underground laboratory experiments, such as LUX [4] and Panda-X [5], astrophysical observations, or LHC experiments to support the existence of exotic dark matter particles.

An alternative way to explain the observed dynamics of galaxies and clusters is to adopt a modified theory of gravitation. Modified Newtonian dynamics (MOND) [6, 7] uses an empirical non-relativistic formula for gravitational acceleration to fit observed galaxy velocity profiles. MOND combines a characteristic minimum acceleration scale $a_{0}=1.2 \times$ $10^{-10} \mathrm{~m} / \mathrm{s}^{2}$ with empirical choice from many possible interpolating functions. But it is not based on a covariant fundamental action principle and MOND fails to fit clusters and cosmology without dark matter.

A more satisfactory modified gravitational theory would fulfil the following requirements:

Email addresses: mgreen@perimeterinstitute.ca (M. A. Green), jmoffat@perimeterinstitute.ca (J. W. Moffat)

(C) 2019. This manuscript version is made available under the CC-BY-NCND 4.0 license

http://creativecommons.org/licenses/by-nc-nd/4.0/
- general covariance and local Lorentz invariance

- classical causal locality

- equivalence principle

- contains General Relativity (GR) in a well-defined limit.

Modified gravity (MOG), originally called Scalar-Tensor-VectorGravity (STVG), satisfies these four requirements [8]. MOG can explain the dynamics of galaxies, clusters and the largescale structure of the universe without needing to add dark matter. Using phase-space analysis, it was shown in [9] that MOG has a viable sequence of cosmological epochs. MOG has been shown to fit a large number of galaxy rotation curves [10], globular cluster velocity dispersions [11] and lensing observations [12], and ultra-diffuse galaxy NGC 1052-DF2 [13], satisfy the Tully-Fisher relation [14, 15], successfully describe the dynamics of clusters [16] and merging clusters, such as the Bullet Cluster and the Train Wreck Cluster Abell 520 [17, 18], and model the growth of structure to fit the observed matter power spectrum at present [19.-22].

The LIGO/Virgo observatory detection of the merging neutron stars gravitational wave event $G W 170817$, together with the optical detection GRB170817A, has determined that gravitational waves move with the speed of light to one part in $10^{15}$. MOG is also compatible with this observational result [23].

We report here on an investigation of the match between gravitational accelerations calculated using MOG and accelerations inferred from rotational velocities for a diverse set of galaxies. McGaugh et al. [24] (MLS) have demonstrated an empirical radial acceleration relation (RAR) between predicted Newtonian accelerations and accelerations inferred from observations of 153 galaxies in the Spitzer Photometry and Accurate 
Rotation Curves (SPARC) dataset. A characteristic deviation from Newtonian gravitational acceleration is evident for a wide range of galaxy masses and types. In the following, we will demonstrate the ability of MOG to fit accelerations inferred from the same SPARC galaxy data, with suitable allowances for the uncertainties of galaxy parameters.

For each SPARC galaxy, data made available by McGaugh et al ${ }^{1}$ includes the galaxy distance, inclination, $3.6 \mu \mathrm{m}$ surface photometry, total luminosity, $\mathrm{HI}$ mass and radius, and rotational velocity profiles. The provided data also lists the inferred contributions of bulge, stellar disk and gas components to Newtonian rotational velocity at radii corresponding to the rotational velocity measurements. Calculation of these velocity contributions clearly required models for the 3-dimensional mass distributions of bulge, disk and gas within the galaxy; but details were not provided for the mass models used in MLS. Mass models are also needed to calculate MOG accelerations. We have used the surface photometry and other data listed above to develop the needed galaxy mass models.

The next Section introduces the MOG acceleration law for non-relativistic systems with weak fields. It is followed by a summary of analyses of SPARC galaxy data and fits to the RAR reported in MLS and subsequent studies. In Section 4 we describe our independent analysis of the SPARC data, our own fit to the RAR, comparison of MOG predictions to observed accelerations, and adjustment of uncertain galaxy parameters to improve MOG fits. We discuss the significance of the many assumptions and uncertainties involved in analysis and fitting of the galaxy data to empirical or theoretical models and draw conclusions.

\section{MOG Acceleration Law}

Like GR, MOG is a classical theory of gravitation based on a fully covariant action principle and corresponding field equations. In addition to the spacetime metric $g_{\mu \nu}$, the MOG formalism introduced in [8], and summarized in Appendix A, has a massive vector field $\phi_{\mu}$ and non-negative scalar fields $\alpha$ and $\mu$. The MOG gravitational coupling strength is $G=G_{N}(1+\alpha)$, where $G_{N}$ is Newton's gravitational constant. MOG reduces to GR in the limit $\alpha \rightarrow 0$.

The equation of motion for a massive test particle in MOG has the covariant form [8]:

$$
m\left(\frac{d u^{\mu}}{d s}+\Gamma_{\alpha \beta}^{\mu} u^{\alpha} u^{\beta}\right)=q_{g} B^{\mu}{ }_{v} u^{v},
$$

where $u^{\mu}=d x^{\mu} / d s$ with $s$ the proper time along the particle trajectory, $\Gamma_{\alpha \beta}^{\mu}$ denote the Christoffel symbols, and $B_{\mu v}=$ $\partial_{\mu} \phi_{v}-\partial_{\nu} \phi_{\mu}$. Moreover, $m$ and $q_{g}$ denote the test particle mass $m$ and gravitational charge $q_{g}=\sqrt{\alpha G_{N}} m$, respectively. We note that for $q_{g} / m=\sqrt{\alpha G_{N}}$ the equation of motion for a massive test particle (1) satisfies the (weak) equivalence principle, leading to the free fall of particles in a homogeneous gravitational field, although the free-falling particles do not follow geodesics.

${ }^{1}$ Data for the SPARC galaxies was obtained from http://astroweb. cwru.edu/SPARC/
In the weak field region, $r \gg 2 G M$, surrounding a stationary mass $M$ centred at $r=0$ the spherically symmetric field $\phi_{\mu}$, with effective mass $\mu$, is well approximated by the Yukawa potential:

$$
\phi_{0}=-Q_{g} \frac{\exp (-\mu r)}{r}, \quad \phi_{i}=0, i=1 . .3,
$$

where $Q_{g}=\sqrt{\alpha G_{N}} M$ is the gravitational charge of $M$. The radial equation of motion of a non-relativistic test particle, with mass $m$ and at radius $r$, in the field of $M$ is then given by

$$
\frac{d^{2} r}{d t^{2}}+\frac{G M}{r^{2}}=\frac{q_{g} Q_{g}}{m} \frac{\exp (-\mu r)}{r^{2}}(1+\mu r)
$$

The mass $\mu$ is tiny - comparable to the experimental bound on the mass of the photon - giving a range $\mu^{-1}$ of the repulsive exponential term the same order of magnitude as the size of a galaxy. Since $q_{g} Q_{g} / m=\alpha G_{N} M$, the modified Newtonian acceleration law for a point particle can be written as [8]:

$$
a_{\mathrm{MOG}}(r)=-\frac{G_{N} M}{r^{2}}[1+\alpha-\alpha \exp (-\mu r)(1+\mu r)] .
$$

This reduces to Newton's gravitational acceleration in the limit $\mu r \ll 1$.

In the limit that $r \rightarrow \infty$, we get from (4) for approximately constant $\alpha$ and $\mu$ :

$$
a(r) \approx-\frac{G_{N}(1+\alpha) M}{r^{2}} .
$$

MOG has a Newtonian-Kepler behaviour for large $r$ with enhanced gravitational strength $G=G_{N}(1+\alpha)$. The transition from Newtonian acceleration behavior for small $r$ to non-Newtonian behaviour for intermediate values of $r$ is due to the repulsive Yukawa contribution in (4). This can also result in the circular orbital rotation velocity $v_{c}$ having a maximum value in the transition region. The prediction of Newton-Kepler-like rotation curves at large $r$, with $G>G_{N}$, distinguishes MOG from the MOND asymptotic behavior. A first verification of the MOG asymptotic behavior as $r \rightarrow \infty$ has been obtained from a fitting of Milky Way galaxy rotation curves with a distance scale $R \sim 200 \mathrm{kpc}[25]$.

For a distributed baryonic matter source, the MOG (weak field) acceleration law becomes [10]:

$$
\begin{aligned}
\boldsymbol{a}_{\mathrm{MOG}}(\mathrm{x})= & -G_{N} \int d^{3} \mathrm{x}^{\prime} \frac{\rho_{\mathrm{bar}}\left(\mathrm{x}^{\prime}\right)\left(\mathrm{x}-\mathrm{x}^{\prime}\right)}{\left|\mathrm{x}-\mathrm{x}^{\prime}\right|^{3}} \\
& {\left[1+\alpha-\alpha \exp \left(-\mu\left|\mathrm{x}-\mathrm{x}^{\prime}\right|\right)\left(1+\mu\left|\mathrm{x}-\mathrm{x}^{\prime}\right|\right)\right], }
\end{aligned}
$$

where $\rho_{\text {bar }}$ is the total baryon mass density. In the following, $a_{\mathrm{MOG}}, a_{\mathrm{obs}}$ and $a_{\mathrm{bar}}$ will denote the magnitudes of the accelerations indicated by the suffix.

Equations (3) to (6) were derived using the assumption that variations of $\alpha$ and $\mu$ are ignorably small within the spacetime region being considered. In this paper we adopt a simplified version of MOG that formalizes this assumption. Instead of Eq. (A-4) we set $S_{S}=0$, and treat $\alpha$ and $\mu$ as parameters that run, taking effectively constant values that depend on the scale of the system under investigation and the spatial resolution with which it is observed. This is analogous to the running of masses and coupling parameters associated with renormalization group (RG) flow when a condensed matter or particle physics system is observed at different scales. 


\section{The Radial Acceleration Relation}

Data for 153 galaxies from the SPARC sample of galaxies [26] was used in MLS to demonstrate correlation between the Newtonian acceleration $a_{\text {bar }}$, due to the apparent baryonic matter, and the acceleration $a_{\text {obs }}$ inferred from galaxy rotational velocity curves. Fig. 3 of MLS shows, as a histogram of 2693 individual data points for 153 different galaxies, results that demonstrate obvious correlation between $g_{\text {obs }}$ (our $a_{\text {obs }}$ ) and $g_{\text {bar }}\left(\right.$ our $\left.a_{\text {bar }}\right){ }^{2}$ In MLS, a RAR that fits the empirical data is given by

$$
a_{\mathrm{obs}}=\mathscr{F}\left(a_{\mathrm{bar}}\right)=\frac{a_{\mathrm{bar}}}{1-\exp \left(-\sqrt{a_{\mathrm{bar}} / a_{0}}\right)},
$$

where $a_{0}=(1.20 \pm 0.02($ random $) \pm 0.24$ (systematic $\left.)\right) \times 10^{-10}$ $\mathrm{m} \mathrm{sec}^{-2}$. The random error is a $1 \sigma$ value, while the $20 \%$ systematic uncertainty is attributed to normalization uncertainty of the stellar mass-to-light ratio. Eq. (7) contains the one critical acceleration scale $a_{0}$; for $a_{\mathrm{bar}} \gg a_{0}$ it gives $a_{\mathrm{obs}} \approx a_{\mathrm{bar}}$ and for $a_{\mathrm{bar}} \ll a_{0}$ it gives $a_{\mathrm{obs}} \approx \sqrt{a_{\mathrm{bar}} a_{0}}$.

The results of MLS were obtained using best estimated values for galaxy inclination, $i$, and galaxy distance, $D$. Mass models were derived from $3.6 \mu \mathrm{m}$ images and HI profiles. The HI data was compiled from other studies and made available in the SPARC database, without uncertainties, as total HI mass $M_{\mathrm{HI}}$ and radius $R_{\mathrm{HI}}$ where $\mathrm{HI}$ surface density (corrected to face-on) reaches $1 M_{\odot} \mathrm{pc}^{-2}$. Generic functional forms were assumed for bulge and stellar disk components; but algorithmic details of the decomposition into two or three (including bulge) components and transformation from the inclined line-of-sight (LoS) to face-on (FO) were not provided. The total mass of gas was assumed to be $1.33 \mathrm{M}_{\mathrm{HI}}{ }^{3}$ Disk and bulge mass-to-light ratios were assumed to have the fixed values $\Upsilon_{\text {disk }}=\Upsilon_{\star}=0.5 M_{\odot} / L_{\odot}$, $\Upsilon_{\text {bul }}=1.4 \Upsilon_{\text {disk }}$. This is supported by analysis presented in [27], which showed the RARs for individual galaxies and investigated the effect of choosing different fixed values for $\Upsilon_{\text {bul }}$ and $\Upsilon_{\text {disk }}$. Plots in [27] show that for many individual galaxies, especially those with $M_{\text {bar }}<3 \times 10^{10} \mathrm{M}_{\odot}$, the observed accelerations deviate greatly from the functional form (7) with a fixed value of $a_{0}$.

Choosing fixed $a_{0}=1.20 \times 10^{-10} \mathrm{~m} \mathrm{sec}^{-2}$, Li et al. [28] fit the RAR (7) to individual galaxies by marginalizing over $\Upsilon_{\star}$, $i$ and $D$, with Gaussian priors whose widths match estimated observational errors. They did similar fits with $a_{0}$ added to the list of adjustable parameters; separate analyses were done assuming a prior for $a_{0}$ that is flat from 0 to $10^{-9} \mathrm{~m} \mathrm{sec}^{-2}$, and a Gaussian prior with $a_{0}=(1.20 \pm 0.02) \times 10^{-10} \mathrm{~m} \mathrm{sec}^{-2}$. As one should expect, use of the best fit parameters yields histograms with much less dispersion about the RAR curve than in Fig. 3 of MLS.

\footnotetext{
${ }^{2}$ Exclusion of rotational velocity data points with $\leq 10 \%$ uncertainty means that only 147 galaxies actually contribute to Fig. 3 of MLS. The present work excludes points with $<10 \%$ uncertainty, thus keeping 7 more data points and increasing the number of galaxies to 149 .

${ }^{3}$ The contributions, $v_{\text {gas }}$, of each galaxy's gas to rotational velocity reflect the compiled HI profiles, which were not included with the SPARC data and not used in the present work.
}

Fig. 7 of [28] shows that, in the above case of $a_{0}$ with a flat prior, the distribution of optimal $a_{0}$ values has a full width of about an order of magnitude. Rodrigues et al. [29], marginalizing over $\Upsilon_{\text {disk }}, \Upsilon_{\text {bul }}, D$ and $a_{0}$ but not $i$ found a similarly broad distribution. From the significant variation and uncertainty of fitted $a_{0}$ values, they conclude that the "emergent acceleration" obtained by combining the results from individual galaxies "cannot be considered a fundamental acceleration".

\section{Analysis of SPARC data with MOG}

The "mass models" made available by McGaugh et al. for the SPARC galaxies specify the face-on surface density only at radii for which velocity measurements are available. Since calculation of MOG accelerations, using Eq. (6), requires knowledge of $\rho_{\text {bar }}$ at all $(r, z)$ within a galaxy, we used the given data, excluding rotational velocities, to independently develop the needed mass models.

Decomposition of the $3.6 \mu \mathrm{m}$ photometry observations into bulge and disk surface brightness radial profiles is described in [26]. Bulges were identified for 32 galaxies; the remainder were assumed to have disks only. Our independent analysis of the photometry data, to determine and characterize bulge and disk components, identified bulges for 57 of 149 galaxies. Exponentials were fitted to the outer disk and used to extrapolate to larger radii. Bulges were assumed to be spherical. Stellar disks were assumed to have a $\operatorname{sech}^{2}$ vertical mass distribution [30, 31] with scale height $z_{\mathrm{d}}=0.196 R_{\mathrm{d}}{ }^{0.633}$, where $R_{\mathrm{d}}$ is the given scale length in kpc [32].

We converted the observed stellar disk surface brightness profiles $\Sigma_{\text {disk,obs }}(r)$ to equivalent face-on profiles $\Sigma_{\text {disk,FO }}(r)$ by numerically integrating along the line-of-sight, with inclination $i$, through a provisional stellar density model $\rho_{\text {disk }}(r, z)$ to determine the corresponding model line-of-sight surface brightness $\Sigma_{\text {disk,LoS }}(r)$. Keeping the $z$ dependence fixed, $\rho_{\text {disk }}$ was then iteratively adjusted to make the ratio $\Sigma_{\text {disk,LoS }}(r) / \Sigma_{\text {disk,obs }}(r)$ converge to 14 This gave the 3 -d stellar disk density. A similar iterative process was used to determine the bulge radial density $\rho_{\text {bul }}$ from the bulge surface brightness $\Sigma_{\text {bul,obs }}(r)$. Plots of the bulge-disk decompositions and derived face-on models are provided in the Supplementary Material.

Instead of the fixed ratio 1.33, we chose gas disk models that reflect a variation of $\eta=M_{\text {gas }} / M_{\mathrm{HI}}$ with galaxy morphological type, where $M_{\text {gas }}$ is the total gas mass. This allows for typical abundances of gases other than $\mathrm{HI}$ and $\mathrm{He}$, for which there was no data or modeling allowance in the analysis of MLS. We also incorporated depressions in the central regions [33]. Our gas density models do not consider the radial variation of the ratio of HI to other gases, nor do they have the benefit of observed HI profiles. Actual total gas density profiles are likely to have large statistical variations (as much as 1 dex for early-type galaxies) about these models [34]. For the ratio $\eta$ we used a relation from

\footnotetext{
${ }^{4}$ The iteration was started by treating the observed profile $\Sigma_{\text {disk,obs }}(r)$ as face-on, with vertical mass distribution as described above.
} 
[35] based on the numerical morphological type $T$ :

$$
\eta(T)=\left\{\begin{array}{l}
1.4\left(4.7-0.8 T+0.043 T^{2}\right), T<9, \\
1.4, \quad T \geq 9
\end{array}\right.
$$

These values range from $\eta(0)=6.58$ to $\eta(9)=1.4$, and increase the average total mass of the 153 studied galaxies by 15 percent when compared to masses calculated with $\eta=1.33$. The gas disk was assumed to have the same effective scale height as the stellar disk. A radial exponential form with scale length $r_{g}$ was assumed at large radius, but this was depressed in the central region by the factor

$$
f(r)=\left[1-\frac{1}{\eta(T)}\left(1+\frac{r_{g}}{R_{\mathrm{d}}}\right)^{-2}\right]\left(1-\frac{1}{\eta(T)} \exp \left(-r / R_{\mathrm{d}}\right)\right) .
$$

The factor in square brackets is for normalization: the central gas density and $r_{g}$ were chosen to yield the total gas mass $M_{\text {gas }}=\eta(T) M_{\mathrm{HI}}$ and the given $R_{\mathrm{HI}}$ at modeled HI surface density of $1 \mathrm{M}_{\odot} / \mathrm{pc}^{2}$.

The relation of Newtonian gravitational acceleration $a_{\text {Newton }}$ (calculated using our new mass models) to the centripetal acceleration $a_{\mathrm{obs}}$ (inferred from observed rotational velocities, $v_{\mathrm{obs}}$ ) is shown in Fig. 1. The histogram is similar to that of Fig. 3 of MLS, with about 30 percent less discrepancy between $a_{\text {Newton }}$ and $a_{\mathrm{obs}}$. In Appendix $\mathrm{B}$ we show that the reduced discrepancy arises from the increased mass of gas in our models. The Supplementary Material includes plots for each galaxy of observed and calculated Newtonian rotation curves (with stellar and gas components). Whether the mass models of McGaugh et al. or our mass models are more representative of reality will require more information to decide. Qualitatively they give similar results, so use of our mass models to calculate MOG accelerations seems reasonable.

For a given mass density model $\rho_{\text {bar }}(r, z)$, MOG accelerations can be computed using Eq. (6) and numerical integration. For the running MOG parameters $\mu$ and $\alpha$ we have used the functional forms [36]:

$$
\mu(M)=\frac{D_{0}}{\sqrt{M}}, \quad \alpha(M)=\alpha_{\infty} \frac{M}{\left(\sqrt{M}+E_{0}\right)^{2}},
$$

where $M$ is the total mass of the galaxy, $\alpha_{\infty}=10, D_{0}=6.25 \times$ $10^{3} \sqrt{M_{\odot}} / \mathrm{kpc}$ and $E_{0}=2.5 \times 10^{4} \sqrt{M_{\odot}}$. Eqs. 10 were derived from a point source solution of the MOG field equations and should thus be considered only approximately valid for mass distributed as in a galaxy. The given values of $\alpha_{\infty}, D_{0}$ and $E_{0}$, determined by fitting to a small sample of galaxies, are subject to adjustment as warranted by additional data; but they are used here without change. Comparison of the calculated MOG gravitational acceleration $a_{\mathrm{MOG}}$ with the centripetal acceleration $a_{\mathrm{obs}}$ is shown in Fig. 2 .

In spite of the global correlation demonstrated in Fig. 3 of MLS and Fig. 1, substantial qualitative and quantitative differences exist for many individual galaxies between the observed rotational velocity curves and velocity curves calculated using the RAR relation (7), or Eq. (6) for MOG. These differences

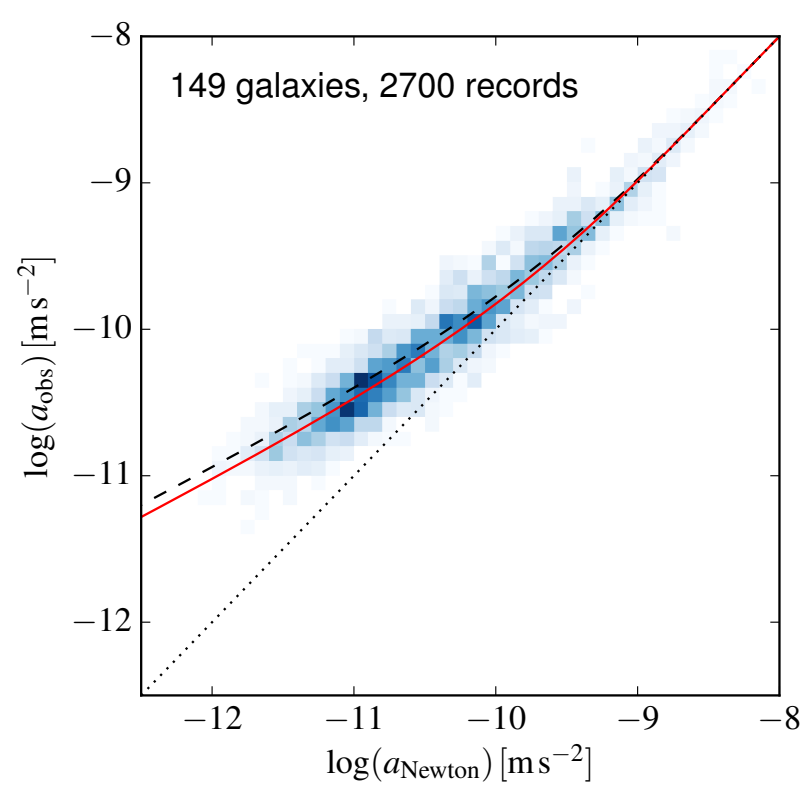

Figure 1: Relation of centripetal acceleration $a_{\mathrm{obs}}$ to Newtonian acceleration $a_{\text {Newton}}$, based on our new mass models. The dashed black curve is the RAR 77, with $a_{\text {bar }}=a_{\text {Newton }}$ and $a_{0}=1.2 \times 10^{-10} \mathrm{~m} / \mathrm{s}^{2}$. The solid red curve is the RAR using $a_{0}=(8.1 \pm .6) \times 10^{-11} \mathrm{~m} / \mathrm{s}^{2}$, which is the mean $\pm 1 \sigma$ of the best fit values for individual galaxies. The fit for each galaxy weights data points inversely with both the fractional uncertainties of $a_{\mathrm{obs}}$ and the local density of points in the radial direction. Closely spaced $v_{\mathrm{obs}}$ records are thus prevented from dominating over more widely spaced records. Similarly weighted residuals relative to the RAR curve have a width $\sigma=0.14 \mathrm{dex}$, compared with $\sigma=0.11$ dex in Fig. 3 of MLS.

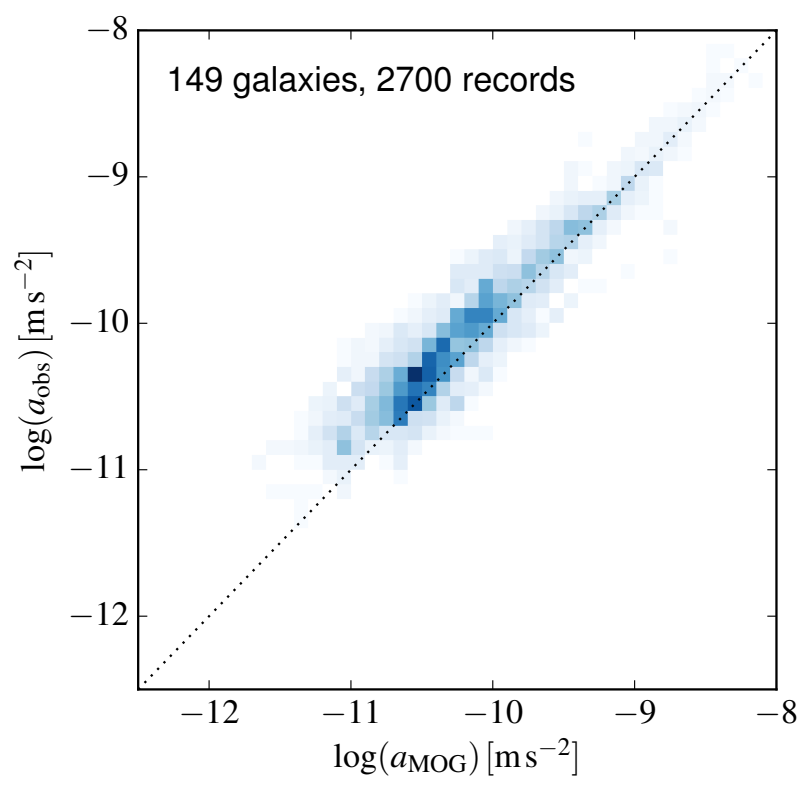

Figure 2: Relation of centripetal acceleration $a_{\mathrm{obs}}$ to MOG acceleration $a_{\mathrm{MOG}}$, using the same galaxy parameters as Fig. 11 The mean $\left\langle\log \left(a_{\mathrm{obs}} / a_{\mathrm{MOG}}\right)\right\rangle=$ $0.13 \mathrm{dex}$, and the residuals have width $\sigma=0.21 \mathrm{dex}$.

will be due, in part, to limitations of the underlying data, discrepancies between the data and true values and, for MOG, the need to adjust $\alpha_{\infty}, D_{0}$ and $E_{0}$. For example, the SPARC 


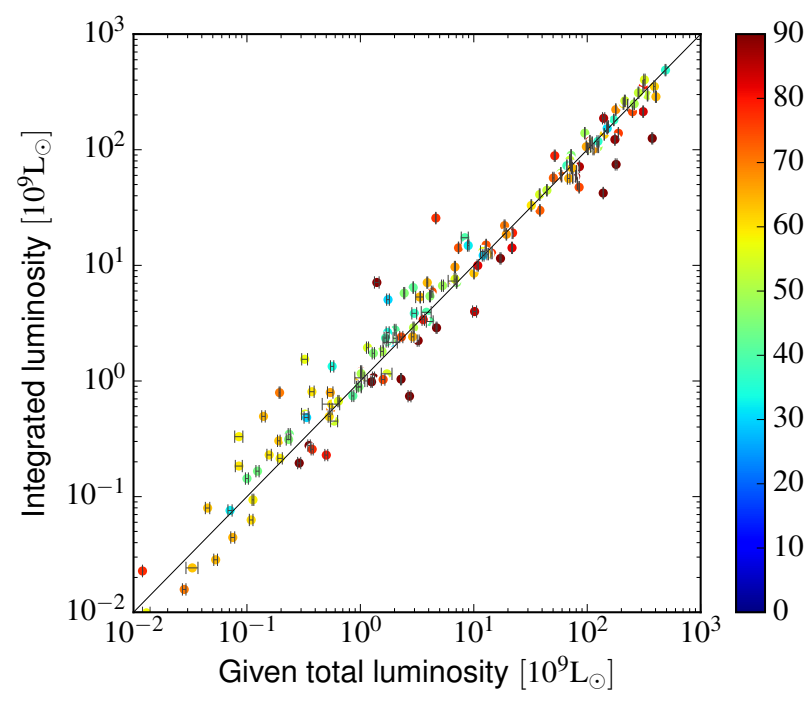

Figure 3: Comparison of given total luminosities $L_{[3.6]}$ with integrated luminosities $L_{\text {mod }}$ for 153 galaxies. Error bars show the given uncertainties, $\delta L_{[3.6]}$. Color indicates the galaxy inclination angle in degrees.

data includes values for total luminosity $L_{[3.6]}$, the uncertainties $\delta L_{[3.6]}$, and stellar luminosity profiles $\Sigma_{\text {obs }}(r)$. From the latter, we have determined model face-on profiles $\Sigma_{\text {mod,FO }}(r)=$ $\Sigma_{\text {disk,FO }}(r)+\Sigma_{\text {bul,FO }}(r)$, which we integrated to obtain total luminosities:

$$
L_{\mathrm{mod}}=\int_{0}^{4.5 R_{\mathrm{d}}} \Sigma_{\bmod , \mathrm{FO}}(r) 2 \pi r d r
$$

Fig. 3 compares values of $L_{[3.6]}$ to $L_{\text {mod }}$. Differences $\Delta L=$ $L_{\text {mod }}-L_{[3.6]}$ generally far exceed the $\pm \delta L_{[3.6]}$ error bars. Explaining these discrepancies does not seem feasible without information, that has not been provided, about how the given $L_{[3.6]}$ and surface brightness profiles were derived from the 2-d galaxy images. Further discussion and analysis of this issue is provided in Appendix B

Errors in several scalar parameters $\Theta$ will affect the accuracy of galaxy mass models and the match between observed rotational velocities, $v_{\text {obs }}$, and predicted velocities, $v_{\text {th }}$, based on a chosen theory and the modeled $\rho_{\Theta}(r, z)$. Besides the total luminosity, discussed above, these parameters include galaxy distance, $D$, inclination, $i$, disk mass-to-light ratio, $\Upsilon_{*}$, the ratio $\Upsilon_{\text {bul }} / \Upsilon_{*}$, and total gas mass, $M_{\text {gas }}=\eta(T) M_{\mathrm{HI}}$. Limiting the range to \pm 1 -sigma from the given values, we varied these parameters to obtain the best fits of $v_{\mathrm{MOG}}(r)$ to $v_{\mathrm{obs}}(r)$. In the fits, observed velocities were weighted by $v_{\mathrm{obs}}(r) / \delta v_{\mathrm{obs}}(r)$. For $D$ and $i$ we used the given uncertainties. For luminosity uncertainty, we combined in quadrature the given $\delta L_{[3.6]}, \Delta L$, and $0.2 L_{[3.6]}$, where the last term is a surrogate for $20 \%$ uncertainty of $\Upsilon_{*}$. We constrained the ratio $\Upsilon_{\text {bul }} / \Upsilon_{*}$ to the range $(1.1,1.8)$ and allowed for $20 \%$ uncertainty of $M_{\text {gas }}$. The top panel of Fig. 4 compares $a_{\mathrm{obs}}$ to $a_{\mathrm{MOG}}$ using galaxy parameters fitted as described above, using MOG as the theory. The bottom panel of Fig. 4 compares $a_{\text {obs }}$ to $a_{\text {RAR }}$, calculated using Eq. (7) with the same galaxy parameters (adjusted to fit MOG) and the value $a_{0}=(5.4 \pm .3) \times 10^{-11} \mathrm{~m} / \mathrm{s}^{2}$ that best fits the RAR to the ad-
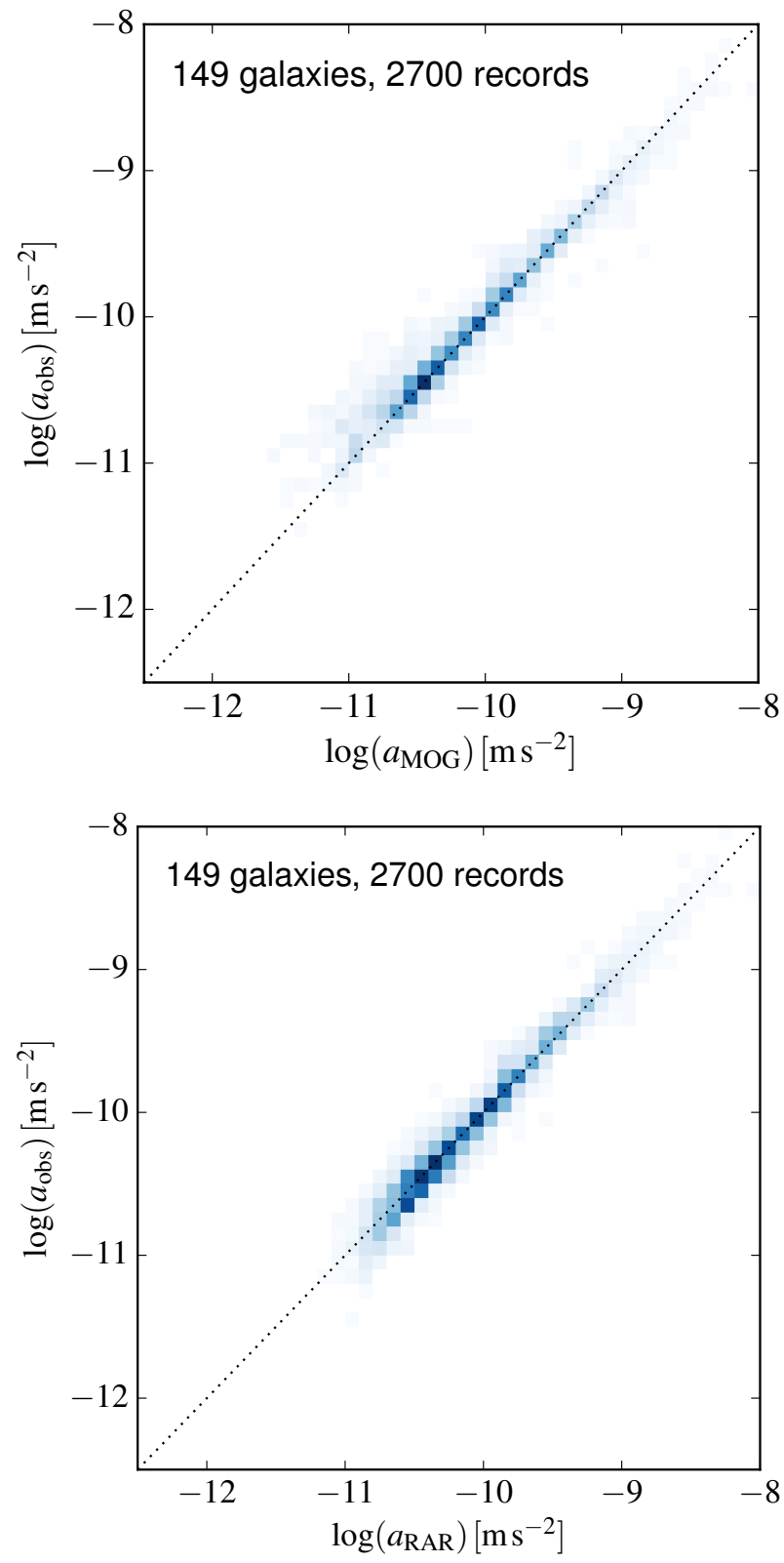

Figure 4: Top panel: Calculated $a_{\mathrm{MOG}}$ accelerations on the horizontal axis, similar to Fig. 2 but with galaxy parameters adjusted as described in the text. The mean $\left\langle\log \left(a_{\mathrm{obs}} / a_{\mathrm{MOG}}\right)\right\rangle=0.05$ dex and residuals about the linear fit, which skew upwards at low acceleration, have a width $\sigma=0.13$ dex. Bottom panel: Same galaxy parameters as in top panel, with calculated $a_{\text {Newton }}$ accelerations transformed to $a_{\text {RAR }}$ using $(7)$, with $a_{0}=(5.4 \pm .3) \times 10^{-11} \mathrm{~m} / \mathrm{s}^{2}$. The mean $\left\langle\log \left(a_{\mathrm{obs}} / a_{\mathrm{RAR}}\right)\right\rangle=-0.006$ dex; residuals have a width $\sigma=0.11$ dex.

justed galaxies. Plots provided in the Supplementary Material show the observed rotational velocity curves and the calculated Newtonian, MOG and RAR predictions, with adjusted parameters, for 153 galaxies.

Fig. 5 shows comparisons of adjusted inclination, distance, integrated luminosity and gas mass to the original, unadjusted values. (The "given" gas masses are $M_{\text {gas }}=\eta(T) M_{\mathrm{HI}}$.) Fig. 6 compares the original and adjusted galaxy total masses. In a great number of cases, the adjustments were limited by our 1- 

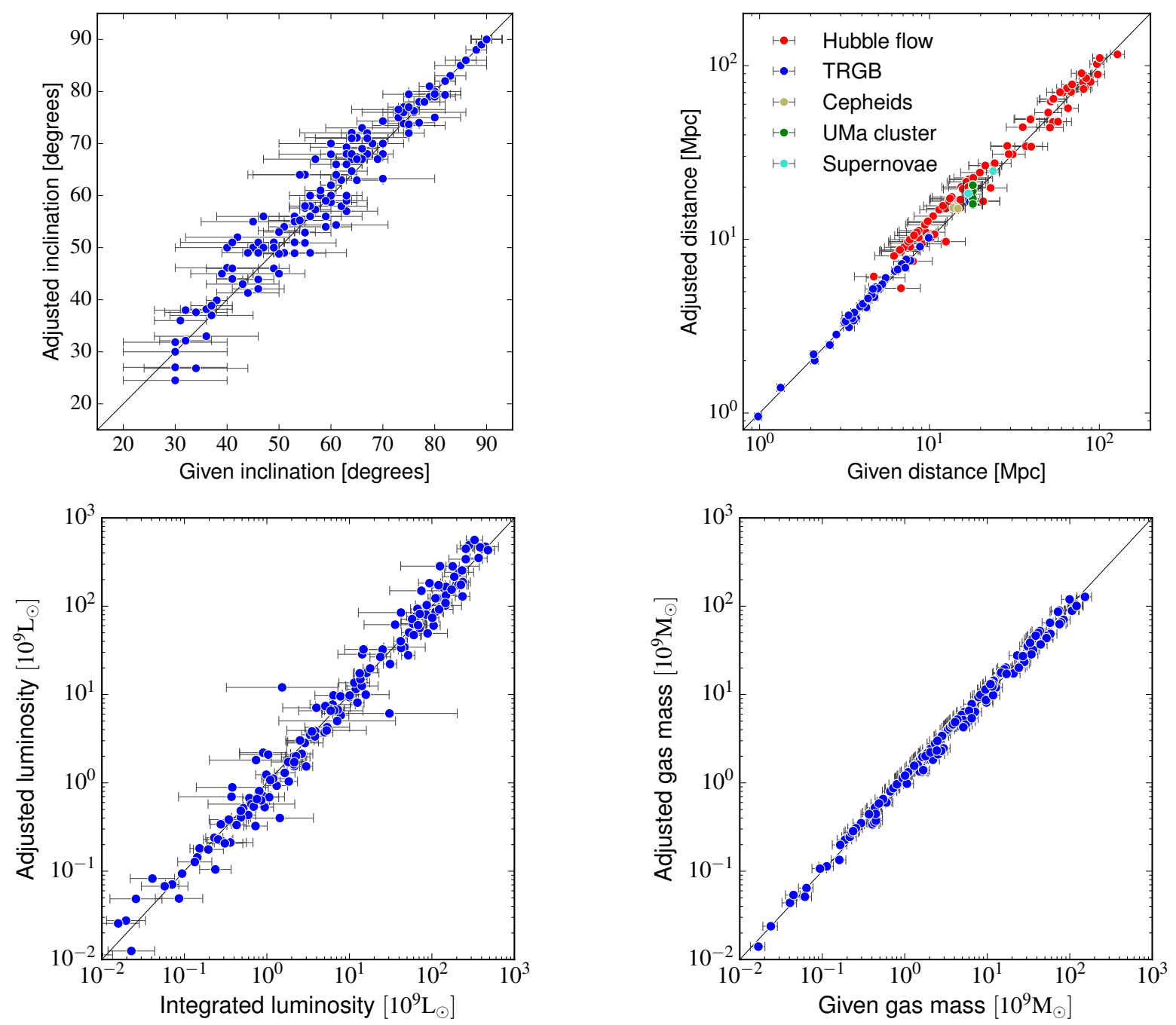

Figure 5: Comparisons of unadjusted to adjusted values of galaxy inclination, distance, integrated luminosity and gas mass. For distances, the legend indicates the method used to determine the given values. Error bars are \pm 1 -sigma, as described in the text.

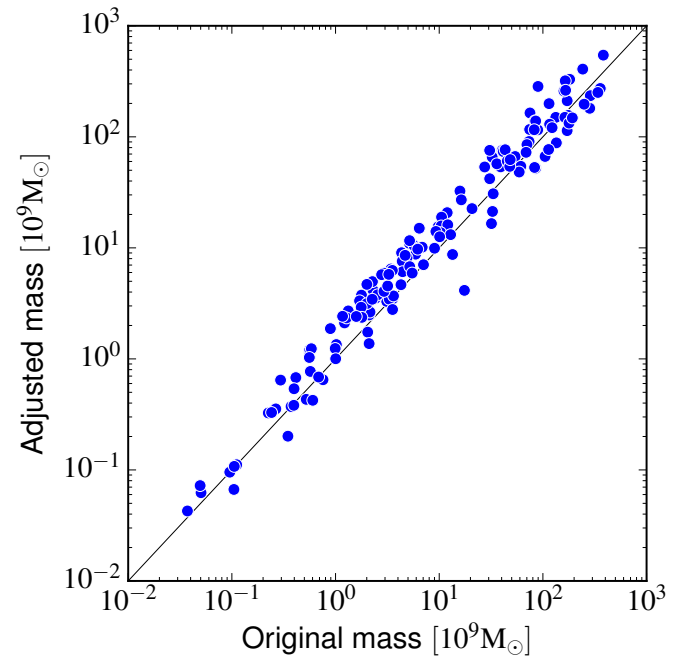

Figure 6: Comparison of original and adjusted total masses. sigma bounds on parameter variations. Nonetheless, the matches between $a_{\mathrm{obs}}$ and both $a_{\mathrm{MOG}}$ and $a_{\mathrm{RAR}}$ in Fig. 4 are quite tight. Similarity of the top and bottom panels of Fig.4 indicates strong correlation of accelerations calculated using either MOG or the RAR.

\section{Discussion}

Many significant uncertainties affected our analysis of the SPARC galaxy data. Uncertainty of total luminosity is highlighted by the discrepancies between $L_{[3.6]}$ and $L_{\text {mod }}$ shown in Fig. 3. the standard deviation of the ratios $L_{\text {mod }} / L_{[3.6]}$ for the 153 selected galaxies is 0.2 dex. Galaxy distances determined using Hubble flow have the greatest uncertainty, with errors of 0.1 dex or more, perhaps systematic, expected at smaller distances due to increased significance of peculiar velocities and local flows. Inferred masses grow quadratically with distance; distance errors affect $a_{\mathrm{obs}}, a_{\mathrm{Newton}}$ and $a_{\mathrm{MOG}}$ differently. The ratio $M_{\mathrm{gas}} / M_{\mathrm{HI}}$ has large uncertainty; and the distribution $\rho_{\mathrm{gas}}$ 
of all gases cannot be reliably inferred from the HI radial profile. Stellar [3.6] mass-to-light ratios for individual galaxies have about 0.1 dex uncertainty [37]. Errors in inclination angles will affect estimates of both mass density and rotational velocity.

Bulge, stellar disk and gas disk model profiles are idealizations that ignore bars, spiral arms, disk warps, non-coplanarity of stellar and gas disks, and galaxy interactions or other disruptions. Such irregularities can have $O(1)$ local effects on rotational velocities at small radii and can result in models not reliably representing the mass distribution. Decomposing radial luminosity profiles into bulge and stellar disk contributions involves subjective judgment. Stellar and gas disk scale heights, determined solely by formula instead of observations, have unknown errors that affect the 3-d mass models and calculated radial gravitational accelerations.

Rotational velocity curves were derived from $\mathrm{HI} / \mathrm{H} \alpha$ observations of gas; the assumption that stellar velocities are similar to gas velocities may not be valid if the stellar and gas disks do not have similar thickness or if they are not coplanar. Rotational velocities at small radius will be uncertain because different depths along the line-of-sight will be at different radii and angular positions. Rotational velocities are generally more uncertain, and likely to be underestimated, near the galactic center [39].

Some of our modeling assumptions differ significantly from assumptions used in MLS. Appendix B examines the effects of switching to the MLS assumption $\mu=1.33$ and scaling of faceon models to match $L_{[3.6]}$. Fig. B-5 shows that these changes lead to histograms very similar to those of Fig. 4, indicating that these assumptions are not material to conclusions regarding MOG.

The uncertainties listed above, including systematic errors, will undoubtedly have contributed to the dispersion and offset from the diagonal seen in the log-log plots of Figs. 1 and 2 Tuning galaxy parameters to fit a given gravitational acceleration model, such as the RAR (7) or MOG acceleration (6), will, by design, reduce the dispersion and make results more closely match the fitted model. Thus Fig. 4 of [28] shows a very tight distribution about the RAR when $\Upsilon_{*}, D$, and $i$ are optimized; and the top panel of our Fig. 4 shows a tight match between $a_{\mathrm{obs}}$ and $a_{\mathrm{MOG}}$.

In [28], also optimizing $a_{0}$, but imposing a narrow Gaussian prior with mean $1.2 \times 10^{-10} \mathrm{~m} / \mathrm{s}^{2}$, gave the histogram an even tighter distribution. But the broad distribution of optimal $a_{0}$ values obtained when using a flat prior casts doubt on the justification of a narrow prior. We have found, nonetheless, that adjusting galaxy parameters to fit MOG also yields a tight fit to the RAR, with $a_{0}=(5.4 \pm .3) \times 10^{-11} \mathrm{~m} / \mathrm{s}^{2}$. It is shown in Appendix $\mathrm{B}$ that nearly the same value of $a_{0}$ is obtained with modeling assumptions closer to those of MLS.

We have verified that adjusting the MOG parameters $\alpha_{\infty}$, $D_{0}$ and $E_{0}$ of Eq. $[10$, which are fixed for all galaxies, can make the histogram of Fig. 2 and the adjusted mass distribution of Fig. 6 better centred on the diagonal. However, we have not adjusted or optimized the MOG parameters because we believe that the many other uncertainties and modeling assump- tions would seriously diminish the significance of the results. The values of galaxy parameters adjusted to fit MOG depend on the choice of $\alpha_{\infty}, D_{0}$ and $E_{0}$, and would be different for different MOG parameter values.

Perhaps our most surprising result is that galaxy parameters adjusted within $\pm 1 \sigma$ bounds to fit MOG also give a very good match between $a_{\mathrm{obs}}$ and $a_{\mathrm{RAR}}$ as shown in the bottom panel of Fig. 4. This indicates that the MOG acceleration law (6) gives results generically similar to the RAR functional form (7).

\section{Conclusions}

Our analysis has demonstrated that, for 149 SPARC galaxies, adjusting galaxy parameters within \pm 1 -sigma bounds can yield MOG predictions consistent with the given rotational velocity data. A 0.13 dex systematic discrepancy, prior to parameter adjustments, between observed accelerations and MOG predictions can be attributed to uncertainties of galaxy and/or MOG parameters. Considering the uncertainties involved, there is no material discrepancy between MOG and the given SPARC galaxy data. This contrasts with the significant discrepancy between Newtonian gravitational accelerations and observed accelerations demonstrated in [24].

\section{Acknowledgments}

We thank Viktor Toth for helpful discussions and the anonymous referee for valuable feedback. This research was supported in part by Perimeter Institute for Theoretical Physics. Research at Perimeter Institute is supported by the Government of Canada through the Department of Innovation, Science and Economic Development Canada and by the Province of Ontario through the Ministry of Economic Development, Job Creation and Trade.

\section{A. MOG summary}

A detailed introduction to MOG is given in [8]; here we provide a quick summary. The MOG action is given by

$$
S=S_{G}+S_{\phi}+S_{S}+S_{M}
$$

where $S_{M}$ is the matter action and

$$
\begin{aligned}
S_{G}=\frac{1}{16 \pi} \int d^{4} x \sqrt{-g}\left[\frac{1}{G}(R+2 \Lambda)\right], \\
S_{\phi}=\int d^{4} x \sqrt{-g}\left[-\frac{1}{4} B^{\mu v} B_{\mu v}+\frac{1}{2} \mu^{2} \phi^{\mu} \phi_{\mu}\right], \\
S_{S}=\int d^{4} x \sqrt{-g}\left[\frac{1}{G^{3}}\left(\frac{1}{2} g^{\mu v} \partial_{\mu} G \partial_{v} G-V_{G}\right)\right. \\
\left.+\frac{1}{\mu^{2} G}\left(\frac{1}{2} g^{\alpha \beta} \partial_{\alpha} \mu \partial_{\beta} \mu-V_{\mu}\right)\right],
\end{aligned}
$$

where $B_{\mu \nu}=\partial_{\mu} \phi_{v}-\partial_{\nu} \phi_{\mu}$ and $V_{G}$ and $V_{\mu}$ are potentials. Note that we choose units such that $c=1$, and use the metric signature $[+,-,-,-]$. The Ricci tensor is

$$
R_{\mu v}=\partial_{\lambda} \Gamma_{\mu v}^{\lambda}-\partial_{v} \Gamma_{\mu \lambda}^{\lambda}+\Gamma_{\mu \nu}^{\lambda} \Gamma_{\lambda \sigma}^{\sigma}-\Gamma_{\mu \lambda}^{\sigma} \Gamma_{v \sigma}^{\lambda} .
$$


The matter stress-energy tensor is obtained by varying the matter action $S_{M}$ with respect to the metric:

$$
T_{M}^{\mu v}=-2(-g)^{-1 / 2} \delta S_{M} / \delta g_{\mu v} .
$$

Varying $S_{\phi}+S_{S}$ with respect to the metric yields

$$
T_{\mathrm{MOG}}^{\mu v}=-2(-g)^{-1 / 2}\left[\delta S_{\phi} / \delta g_{\mu v}+\delta S_{S} / \delta g_{\mu v}\right]
$$

Combining these gives the total stress-energy tensor

$$
T^{\mu v}=T_{\mathrm{M}}^{\mu v}+T_{\mathrm{MOG}}^{\mu v} .
$$

The MOG field equations are given by

$$
\begin{array}{r}
G_{\mu v}-\Lambda g_{\mu v}+Q_{\mu v}=8 \pi G T_{\mu \nu}, \\
\frac{1}{\sqrt{-g}} \partial_{\mu}\left(\sqrt{-g} B^{\mu v}\right)+\mu^{2} \phi^{v}=-J^{v}, \\
\partial_{\sigma} B_{\mu \nu}+\partial_{\mu} B_{v \sigma}+\partial_{v} B_{\sigma \mu}=0 .
\end{array}
$$

Here, $G_{\mu v}=R_{\mu v}-\frac{1}{2} g_{\mu \nu} R$ is the Einstein tensor and

$$
Q_{\mu \nu}=\frac{2}{G^{2}}\left(\partial^{\alpha} G \partial_{\alpha} G g_{\mu \nu}-\partial_{\mu} G \partial_{\nu} G\right)-\frac{1}{G}\left(\square G g_{\mu \nu}-\nabla_{\mu} \partial_{\nu} G\right)
$$

is a term resulting from the the presence of second derivatives of $g_{\mu v}$ in $R$ in $S_{G}$. The current $J^{v}$, introduced in A-10, is discussed below.

Combining the Bianchi identities, $\nabla_{v} G^{\mu v}=0$, with the field equations $\mathrm{A}-9$ yields the conservation law

$$
\nabla_{v} T^{\mu v}+\frac{1}{G} \partial_{v} G T^{\mu \nu}-\frac{1}{8 \pi G} \nabla_{v} Q^{\mu v}=0 .
$$

It is a key premise of MOG that all baryonic matter possesses, in proportion to its mass $M$, positive gravitational charge: $Q_{g}=\kappa M$. This charge serves as the source of the vector field $\phi^{\mu}$. Moreover, $\kappa=\sqrt{G-G_{N}}=\sqrt{\alpha G_{N}}$, where $G_{N}$ is Newton's gravitational constant and $\alpha=\left(G-G_{N}\right) / G_{N} \geq 0$. Variation of $S_{M}$ with respect to the vector field $\phi^{\mu}$, then yields the MOG 4-current $J_{\mu}=-(-g)^{-1 / 2} \delta S_{M} / \delta \phi^{\mu}$.

For the case of a perfect fluid:

$$
T_{M}^{\mu v}=(\rho+p) u^{\mu} u^{v}-p g^{\mu v},
$$

where $\rho$ and $p$ are the matter density and pressure, respectively, and $u^{\mu}$ is the 4-velocity of an element of the fluid. We obtain from (A-14) and $u^{\mu} u_{\mu}=1$ the 4-current:

$$
J_{\mu}=\kappa T_{M \mu \nu} u^{v}=\kappa \rho u_{\mu} .
$$

It is shown in [38] that, with the assumption $\nabla_{\mu} J^{\mu}=0$, A-13 reduces to

$$
\nabla_{v} T_{M}^{\mu v}=B_{v}^{\mu} J^{v}
$$

It should be stated that in MOG early universe cosmology, using FRW, we do not assume $\nabla_{\mu} J^{\mu}=0[19,23,24]$.

The original formulation of MOG, presented above, also has field equations for $G$ (or $\alpha$ ) and $\mu$ :

$$
\square G=K, \quad \square \mu=L,
$$

where $\square=\nabla^{\mu} \nabla_{\mu}, K=K\left(G, \mu, \phi_{\mu}\right)$ and $L=L\left(G, \mu, \phi_{\mu}\right)$. In the present work, and other recent studies, $\alpha$ and $\mu$ are treated as parameters that are constant for each galaxy, but whose values run with the galaxy mass. With this simplification: $S_{S}=0$, $Q_{\mu v}=0, T_{\mathrm{MOG}}^{\mu v}=-2(-g)^{-1 / 2} \delta S_{\phi} / \delta g_{\mu v}$ and $\nabla_{v} T^{\mu v}=0$.

\section{B. Investigation of modeling differences}

Our development of galaxy mass models $\rho_{\text {bar }}(r, z)$, based on the SPARC data, was described in Section 4 . Some assumptions made in the derivation of these new models differ from apparent and explicit assumptions in MLS. Instead of explicit mass models, the provided SPARC data includes face-on surface brightness profiles $\Sigma_{\text {disk,FO }}(r)$, for radii $r$ at which rotational velocities were also given. How the SPARC $\Sigma_{\text {disk,FO }}(r)$ values were obtained was not specified. We examine here the relationships between line-of-sight and face-on surface brightness profiles from the SPARC data and between equivalent profiles for our new models. We also examine the effects of changing one or both of two significant assumptions made in the derivation of our new models to match those of MLS.

For an axially-symmetric thin disk the line-of-sight and faceon surface brightness should generally satisfy:

$$
R_{\mathrm{FO} / \mathrm{LoS}} \equiv \frac{\Sigma_{\mathrm{disk}, \mathrm{FO}}}{\cos (i) \Sigma_{\mathrm{disk}, \mathrm{LoS}}} \simeq 1
$$

With finite scale height, $z_{d}$, the approximation will fail at radii $r \lesssim z_{\mathrm{d}} / \cos (i)$ because Eq. (B-1) does not consider the exponential disk profile. For nearly edge-on inclinations, $R_{\mathrm{FO} / \mathrm{LoS}}$ will not be useful because $1 / \cos (i)$ diverges.

Fig. B-1 shows plots of $R_{\mathrm{FO} / \mathrm{Los}}$ versus normalized radius $r / r_{d}$ for our new galaxy models (left panel) and as derived directly from the SPARC data (right panel). The plots for the new galaxy models have the expected characteristics; the trend of low inclination galaxies to have $R_{\mathrm{FO} / \mathrm{LoS}}$ slightly below 1 can be attributed to approximation in the iterative determination of $\Sigma_{\text {mod.FO. The plots using }} \Sigma_{\text {disk,FO }}$ and $\Sigma_{\text {disk,LoS }}$ from the SPARC data are not consistent with expectations. Some of the fluctuations can be attributed to limited significant digits of the provided tabular data. Ignoring the quantization noise due to rounding / truncation of the data, especially at large radii, it appears as though the face-on data has been obtained by an almost constant, often substantial, scaling of the line-of-sight data.

Fig. B-2 compares the ratio

$$
R_{[3.6]}=L_{[3.6]} / L_{\bmod }
$$

for the new models of this work to SPARC luminosity ratios, defined as the median value of $R_{\mathrm{FO} / \mathrm{LoS}}$ for each galaxy (as in the right panel of Fig. B-1), that measure the anomalous scaling of $\Sigma_{\text {disk,FO }}(r)$ from the SPARC data beyond the factor of $\cos (i)$. Approximate equality of the $R_{[3.6]}$ and SPARC luminosity ratios suggests that the SPARC $\Sigma_{\text {disk,FO }}$ data were obtained by simply scaling $\Sigma_{\text {disk,LoS }}$ to match the total luminosity, without considering the relation to inclination given by Eq. (B-1). 

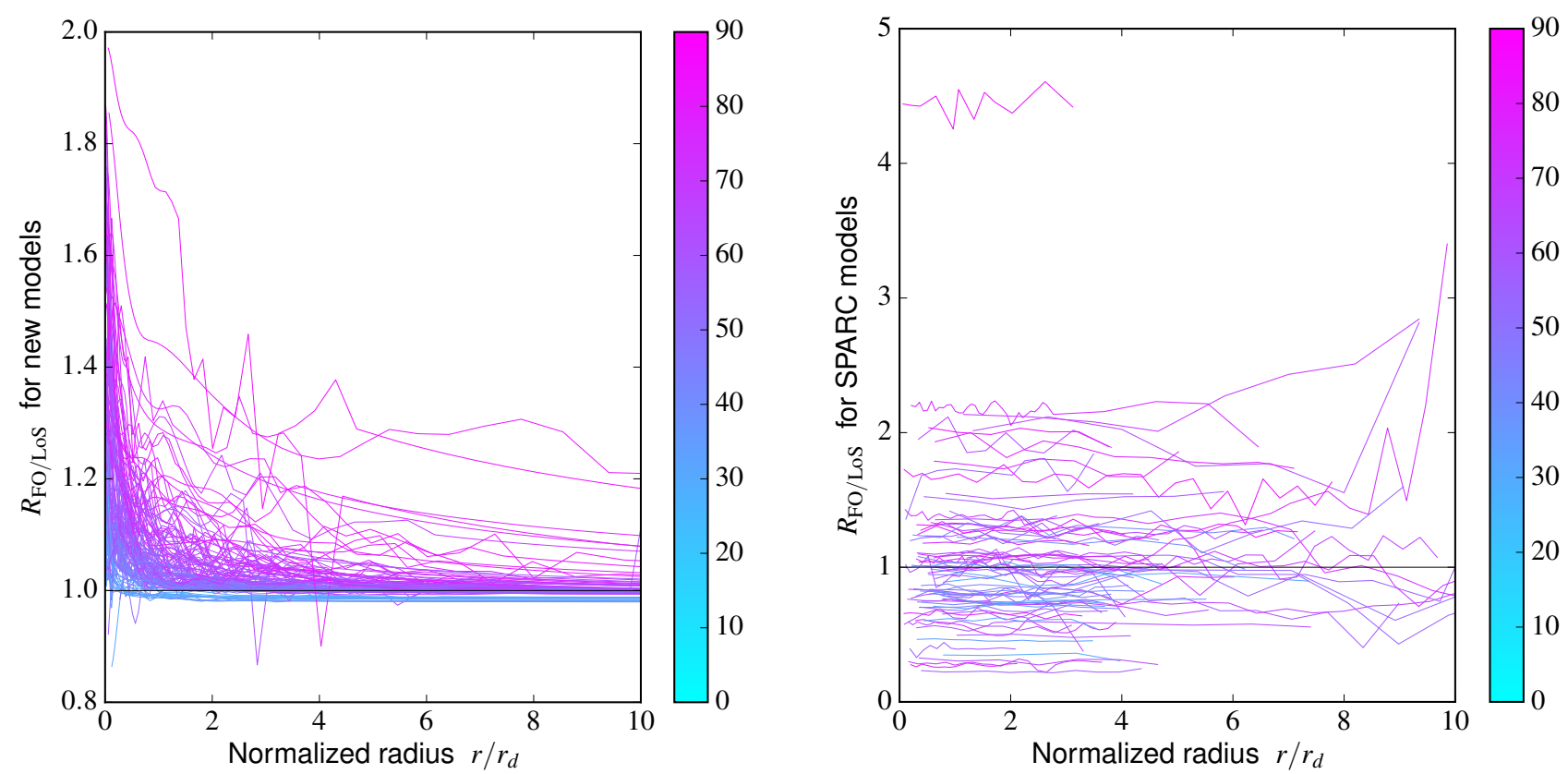

Figure B-1: Left panel: Curves showing $R_{\mathrm{FO} / \text { LoS }}$ for the disks of 135 galaxies with $i \leq 85^{\circ}$, based on the new galaxy models developed as described in Section 4 Colour indicates the galaxy inclination in degrees. Right panel: Similar to left panel, but using the $\Sigma_{\text {disk.FO }}(r)$ values provided with the SPARC data, and showing only 79 galaxies with $i \leq 85^{\circ}$ and for which no bulge was identified in either the present or the SPARC analysis.

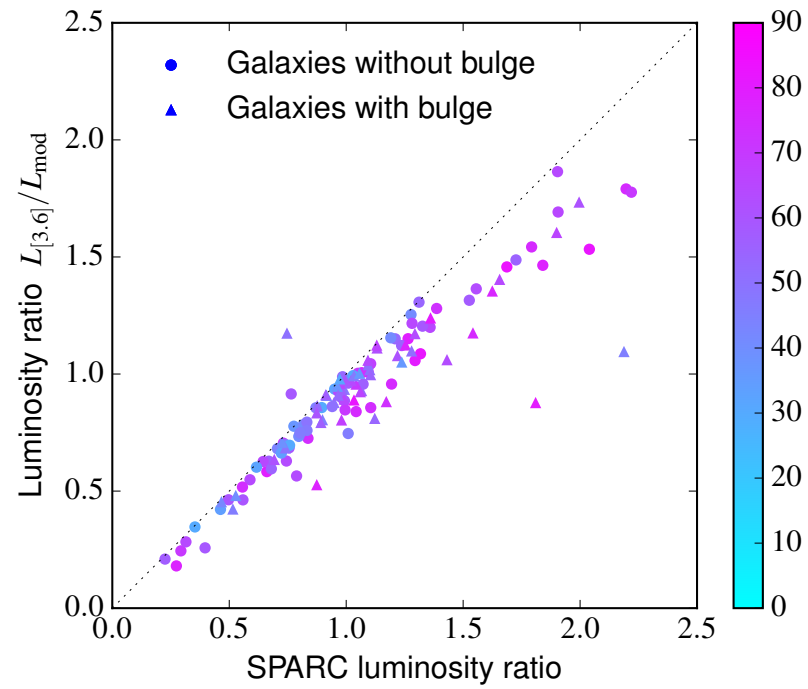

Figure B-2: Comparison of the luminosity ratios $R_{[3.6]}=L_{[3.6]} / L_{\bmod }$ for the new galaxy models to SPARC luminosity ratios, defined as the median of SPARC $R_{\mathrm{FO} / \mathrm{LoS}}$ values (as in the right panel of Fig. $\mathrm{B}-1$, with extreme points excluded. Of the 135 galaxies with $i \leq 85^{\circ}$, bulges were identified in 56 galaxies in our new models and/or the SPARC models.

For our primary analysis, the given surface brightness profiles $\Sigma_{\text {obs }}$ were used as the line-of-sight profiles of axially symmetric galaxies characterized as described in Section 4 However, as shown in Figures 3 and B-2 for many galaxies the total luminosity $L_{\text {mod }}$ obtained from Eq. 11 differs significantly from the given $L_{[3.6]}$. Contributions to the discrepancy between the given and calculated total luminosities will include: inability of profiles, $\Sigma_{\text {obs }}$, along the major axis, to fully represent the irregular 2-d images; and deviations of our (and the SPARC collaboration's) necessarily simple galaxy models from the true, irregular mass distributions.

Scaling $\Sigma_{\text {mod,Fo }}$ in our mass models to make $R_{[3.6]}=1$ is straightforward; the results before any adjustment of galaxy parameters are shown in the left panel of Fig. B-3.

We used Eq. (8) to determine the $M_{\mathrm{gas}} / M_{\mathrm{HI}}$ ratio $\eta$, whereas MLS assumed $\eta=1.33$ for all galaxies. The result of repeating our calculations of $a_{\text {Newton }}$ with $\eta=1.33$ is shown in the right panel of Fig. B-3.

The effects of these revised assumptions can be seen by comparing the histograms of Fig. B-3 with Fig. 11. Scaling

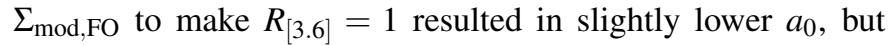
comparable residuals. Setting $\eta=1.33$ resulted in $a_{0}$ that matches the MLS value, with modestly smaller residuals than Fig. 1. Our different assumption regarding gas mass, which can only lead to a larger $M_{\text {gas }}$, seems to be the main reason we found lower $a_{0}$ values than MLS.

The combined effect of scaling $\Sigma_{\text {mod,FO }}$ to make $R_{[3.6]}=1$ and setting $\eta=1.33$ is shown in the left panel of Fig. B-4. The resulting best fit $a_{0}$ is close to the MLS value; the residuals are slightly larger than in our Fig. 1. The right panel shows MOG predictions for these revised mass models, and can be compared with Fig. 2

Using mass models with $R_{[3.6]}=1$ and $\eta=1.33$, and adjusting galaxy parameters using the same algorithm and bounds as used to produce Fig. 4 yields the histograms of Fig. B-5. The best fit value of $a_{0}$ is nearly unchanged from Fig. 4. The residuals of the MOG histogram in Fig. B-5 are slightly increased, while residuals of the RAR histogram are about the same as in the similar histograms of Fig. 4. The great similarity of the 

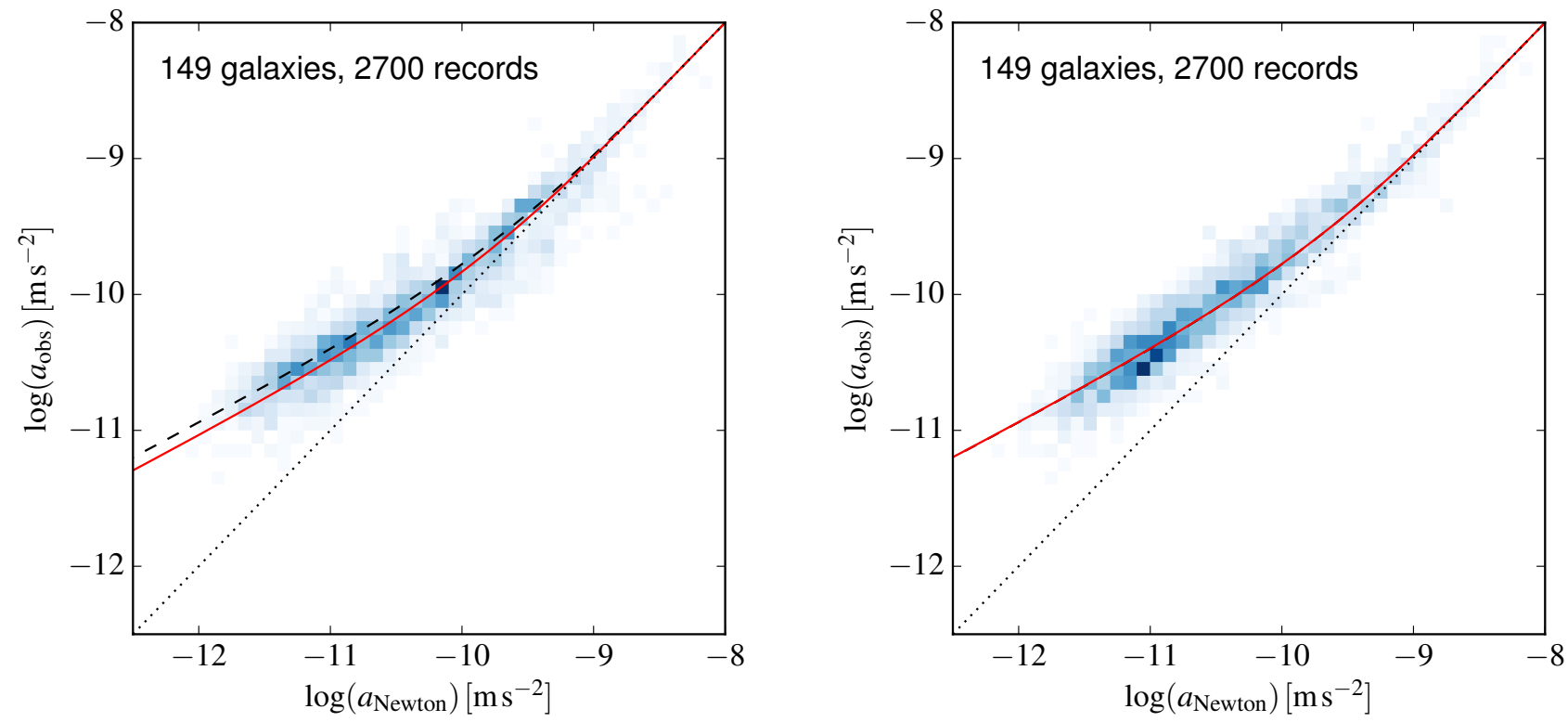

Figure B-3: Left panel: Relation of $a_{\text {obs }}$ to $a_{\text {Newton }}$ when $\Sigma_{\text {mod,FO }}$, for each galaxy, has been scaled to make $R_{[3.6]}=1$. The dashed black curve is the RAR (7) with $a_{0}=1.2 \times 10^{-10} \mathrm{~m} / \mathrm{s}^{2}$. The solid red curve shows the RAR with best fit $a_{0}=(7.6 \pm .6) \times 10^{-11} \mathrm{~m} / \mathrm{s}^{2}$. Residuals relative to the latter curve have a width $\sigma=0.16$ dex. Right panel: Similar to left panel, but with galaxy mass models that, instead of scaling $\Sigma_{\text {mod,FO }}$, have $\mu=M_{\mathrm{gas}} / M_{\mathrm{HI}}=1.33$. The red curve uses the best fit $a_{0}=(1.2 \pm .1) \times 10^{-10} \mathrm{~m} / \mathrm{s}^{2}$. Residuals relative to the red curve have a width $\sigma=0.14 \mathrm{dex}$.
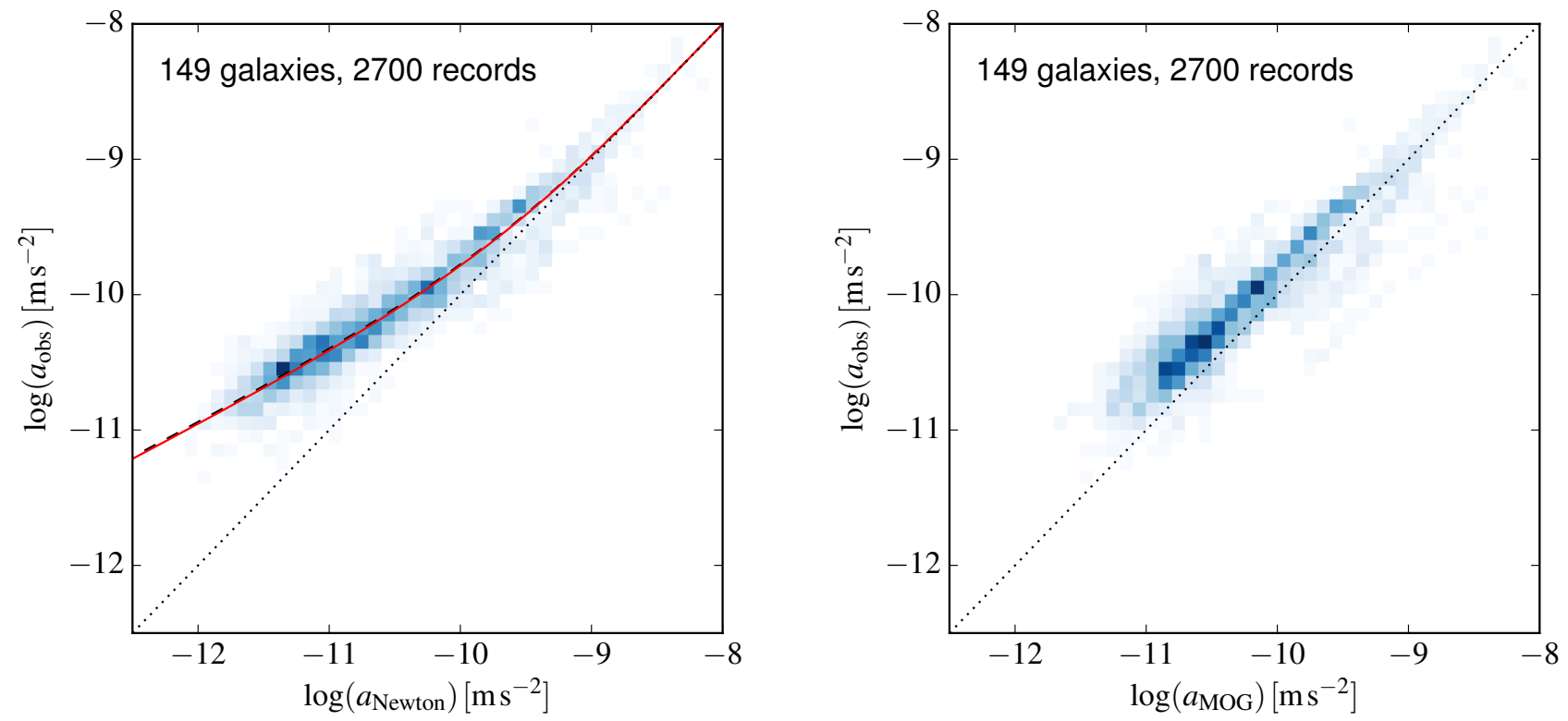

Figure B-4: Left panel: Relation of $a_{\mathrm{obs}}$ to $a_{\text {Newton }}$ with $\Sigma_{\text {mod,FO }}$ scaled to make $R_{[3.6]}=1$ and with $\mu=M_{\mathrm{gas}} / M_{\mathrm{HI}}=1.33$. The dashed black curve is the RAR 7) with $a_{0}=1.2 \times 10^{-10} \mathrm{~m} / \mathrm{s}^{2}$. The solid red curve shows the RAR with best fit $a_{0}=(1.1 \pm .1) \times 10^{-10} \mathrm{~m} / \mathrm{s}^{2}$. Residuals relative to the red curve have a width $\sigma=0.15$ dex. Right panel: Relation of $a_{\mathrm{obs}}$ to $a_{\mathrm{MOG}}$ for the same mass models as the left panel. The mean $\left\langle\log \left(a_{\mathrm{obs}} / a_{\mathrm{MOG}}\right)\right\rangle=0.18$ dex, and the residuals have width $\sigma=0.25$ dex.

histograms of Figs. 4 and B-5 indicates that the ability to find parameters that give good MOG fits is not sensitive to the modeling assumptions. The equally good fit of the adjusted galaxies to the RAR curve, for either set of assumptions, suggests that MOG predictions for galaxy rotation are generically consistent with Eq. (7).

\section{Supplementary Material}

Supplementary Material related to this article can be obtained at https://doi.org/10.1016/j.dark.2019.100323. 

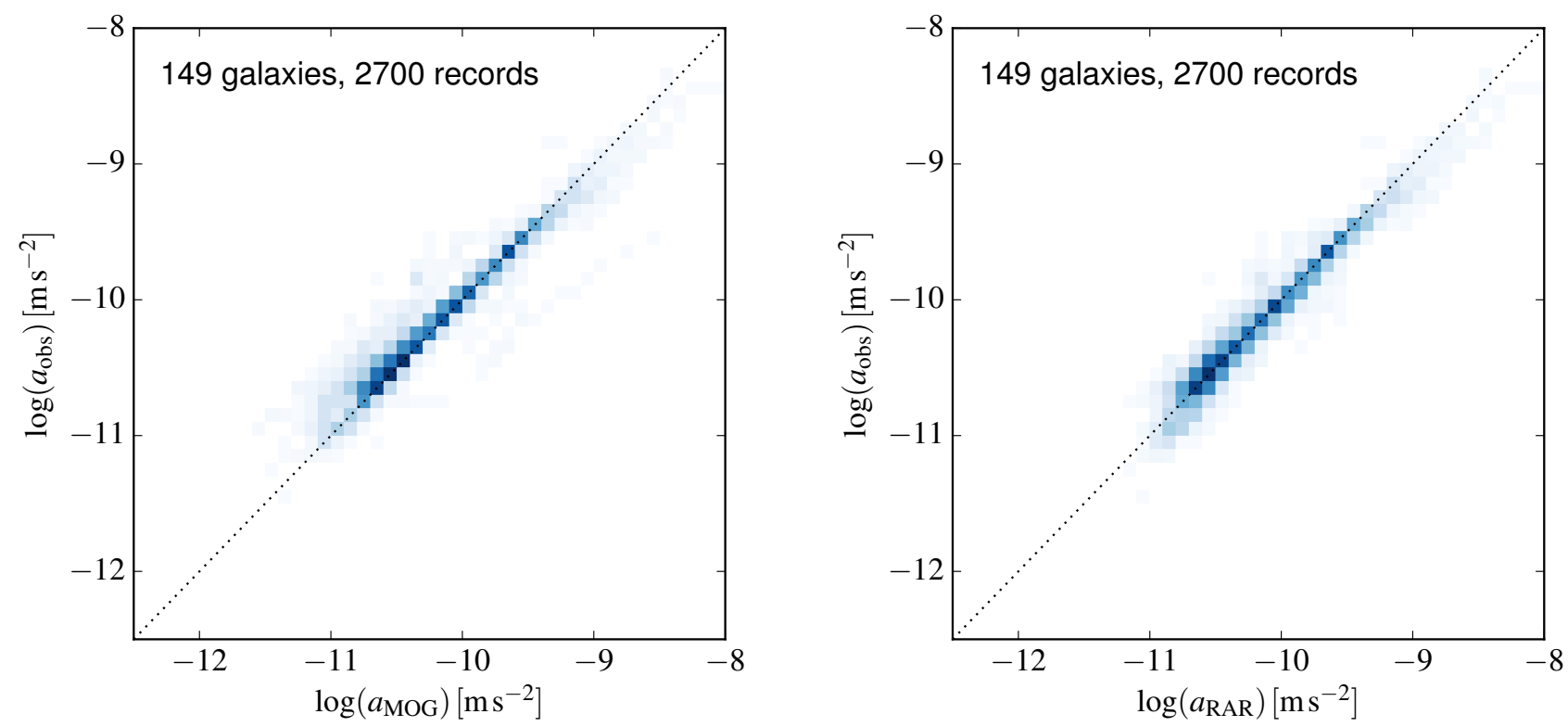

Figure B-5: Left panel: Relation of $a_{\mathrm{obs}}$ to $a_{\mathrm{MOG}}$ for the same mass models as in Fig $\mathrm{B}-4\left(R_{[3.6]}=1, \mu=1.33\right)$ and with galaxy parameters adjusted using the same process as for Fig. 4 The mean $\left\langle\log \left(a_{\mathrm{obs}} / a_{\mathrm{MOG}}\right)\right\rangle=0.06$ dex; residuals have a width $\sigma=0.16$ dex. Right panel: Same galaxy parameters as in top panel, with calculated $a_{\text {Newton }}$ accelerations transformed to $a_{\text {RAR }}$ using 7), with $a_{0}=(5.3 \pm .5) \times 10^{-11} \mathrm{~m} / \mathrm{s}^{2}$. The mean $\left\langle\log \left(a_{\mathrm{obs}} / a_{\mathrm{RAR}}\right)\right\rangle=0.005$ dex; residuals have a width $\sigma=0.11$ dex.

\section{References}

\section{References}

[1] F. Zwicky, Astrophys. J. 886, 217 (1937).

[2] V. C. Rubin and W. K. J. Ford, Astrophys. J. 159, 379 (1970).

[3] V. C. Rubin, N. Thonnard and W. K. J. Ford, Astrophys. J. 225, L107 (1978).

[4] LUX Collaboration: D. S. Akerib et al., Phys. Rev. Lett. 116, 161302 (2016). arXiv:1602.03489 [hep-ex].

[5] PandaX-II Collaboration: A. Tam,, M. Xiao, X. Cui et al., Phys. Rev. Lett. 117, 121303 (2016). arXiv:1607.07400 [hep-ex].

[6] M. Milgrom, Astrophys. J. 270, 371 (1983).

[7] M. Milgrom, arXiv:1609.06642 [astro-ph.GA].

[8] J. W. Moffat, JCAP 0603, 004 (2006). arXiv:gr-qc/0506021

[9] S. Jamali, M. Roshan and L. Amendola, JCAP 01 (2018) 048. arXiv: 1811.04445 [gr-qc].

[10] J. W. Moffat and S. Rahvar, Mon. Not. Roy. Astron. Soc. 436, 1439 (2013). arXiv:1306.6383 [astro-ph.GA].

[11] J. W. Moffat and V. T. Toth, Astrophys. J. 680, 1158 (2008). arXiv:0708.1935 [gr-qc].

[12] J. W. Moffat, S. Rahvar and V. T. Toth, Galaxies, 6, 43 (2018). arXiv: 1204.2985 [gr-qc].

[13] J. W. Moffat and V. T. Toth, Mon. Not. Roy. Astron. Soc.: Letters 482 (2019) L1-L3. arXiv:1805.01117 [gr-qc].

[14] R. B. Tully and J. R. Fisher, Astron. Astrophys. 54, 661 (1977).

[15] M. A. W. Verheijen, Astrophys. J. 563, 694 (2001). arXiv:astro-ph/0108225

[16] J. W. Moffat and S. Rahvar, Mon. Not. Roy. Astron. Soc. 441, 3724 (2014). arXiv:1309.5077 [astro-ph.CO].

[17] J. R. Brownstein and J. W. Moffat, Mon. Not. Roy. Astron. Soc. 382, 29 (2007). arXiv:astro-ph/0702146

[18] N. S. Israel and J. W. Moffat, Galaxies 6, 41 (2018). arXiv:1606.09128 [astro-ph.CO].

[19] J. W. Moffat and V. T. Toth, Galaxies, 1, 65 (2013). arXiv:1104.2957 [astro-ph.CO].

[20] J. W. Moffat, arXiv:astro-ph/0602607

[21] J. W. Moffat, arXiv:1510.07037 [astro-ph.CO].

[22] S. Jamali, M. Roshan and L. Amendola, arXiv:1811.04445 [gr-qc].
[23] M. Green, J. W. Moffat and V. T. Toth, Phys. Lett. B 780, 300 (2018). arXiv: 1710.11177

[24] S. S. McGaugh, F. Lelli and J. M. Schombert, Phys. Rev. Lett. 117, 201101 (2016). arXiv:1609.05917 [astro-ph.GA].

[25] J. W. Moffat and V. T. Toth, Phys. Rev. D91, 043004 (2015). arXiv:1411.6701 [astro-ph.GA].

[26] F. Lelli, S. S. McGaugh and J. M. Schombert, Astronom. J. 152:157 (2016). arXiv:1606.09251 [astro-ph.GA].

[27] F. Lelli, S. S. McGaugh, J. M. Schombert and M. S. Pawlowski, Astrophys. J., 836:152 (2017). arXiv:1610.08981 [astro-ph.GA].

[28] P. Li, F. Lelli, S. McGaugh and J. Schombert. A\&A, 615 (2018) A3. arXiv:1803.00022 [astro-ph.GA].

[29] D. C. Rodrigues, V. Marra, A. del Popolo and Z. Davari, Nat. Astron. 2, 668 (2018). arXiv:1806.06803 [astro-ph.GA].

[30] L. Spitzer, Jr. Astrophys. J., 95:329 (1942).

[31] F. Allaert, G. Gentile, M. Baes et al., A\&A, 582 (2015) A18. arXiv:1507.03095 [astro-ph.GA].

[32] M. A. Bershady, M. A. W. Verheijen, K. B. Westfall et al., Astrophys. J., 716:234 (2010). arXiv:1004.5043 [astro-ph.CO].

[33] J. Wang, J. Fu, M. Aumer et al. MNRAS, 441, 2159 (2014). arXiv:1401.8164 [astro-ph.GA].

[34] A. Boselli, L. Cortese, M. Boquien et al. A\&A, 564, A66 (2014). arXiv:1401.8101 [astro-ph.GA].

[35] S. S. McGaugh and W. J. G. de Blok, Astrophys. J., 481:689 (1997). arXiv:astro-ph/9612070

[36] J. W. Moffat and V. T. Toth, Class. Quantum Grav., 26, 085002 (2009). arXiv:0712.1796 [gr-qc].

[37] S.E. Meidt, E. Schinnerer, G. van de Ven, et al., Astrophys. J., 788:144 (2014). arXiv:1402.5210 [astro-ph.GA].

[38] M. Roshan, Phys. Rev. D87, 044005, (2013). arXiv:1210.3136 [gr-qc].

[39] Y. Sofue and V. Rubin, Ann. Rev. Astron. Astrophys., 39, 137 (2001). arXiv:astro-ph/0010594 